\title{
Perfil de pacientes atendidos por tentativa de suicídio em um Hospital Geral de Emergências do estado de Alagoas, Brasil
}

\author{
Profile of patients treated for attempted suicide at a General Emergency \\ Hospital in the state of Alagoas, Brazil \\ Claudio José dos Santos Júnior ${ }^{1}$ (D), Isaías Vicente Santos ${ }^{2}$ (D), John Victor dos Santos Silva ${ }^{2}$ (D), \\ Valmir de Melo Gomes 3 (D), Mara Cristina Ribeiro 4 (D)
}

\begin{abstract}
RESUMO
Objetivo: Apresentar o perfil dos pacientes atendidos por tentativas de suicídio atendidas pelo Hospital Geral Dr. Oswaldo Brandão Vilela (HGE) da cidade de Maceió, Alagoas, Brasil. Métodos: Trata-se de um estudo do tipo documental, descritivo e retrospectivo com abordagem quantitativa. A amostra da pesquisa foi todo o universo de pacientes atendidos e diagnosticados por tentativa de suicídio no HGE no período de 2015 a 2017. Resultados: A amostra investigada foi constituída de 824 usuários, 50,1\% dos pacientes tem a faixa etária entre 15 a 29 anos e $63 \%$ são do sexo feminino, $82,1 \%$ utilizou o meio de envenenamento, desses, $58,9 \%$ utilizou o agente medicamento. Conclusão: De 2015 a 2017 a distribuição de ocorrências por atendimentos de suicídio cresceu ao longo dos anos entre os jovens. Infere-se que são necessárias ações que permitam e incentivem a prevenção desse dano à população.
\end{abstract}

Palavras-chave: Psiquiatria Comunitária; Saúde Mental; Saúde Pública.

\begin{abstract}
Objective: Presenting the profile of patients treated for suicide attempts at the Oswaldo Brandão Vilela General Hospital (HGE) in the city of Maceió, Alagoas, Brazil. Methods: This is a documentary, descriptive, and retrospective study with a quantitative approach. The research sample was the entire universe of patients assisted and diagnosed at the HGE for suicide in the period from 2015 to 2017 . Results: The sample investigated consisted of 824 users, $50.1 \%$ of the patients had the age range between 15 to 29 years and $63 \%$ are female, $82.1 \%$ used the means of poisoning, of these, 58.9\% used the drug agent. Conclusion: From 2015 to 2017, the distribution of occurrences due to suicide calls increased among young people. It is inferred that actions are necessary that allow and encourage the prevention of this damage to the population.
\end{abstract}

Keywords: Community Psychiatry; Mental Health; Public Health.

1. Acadêmico de medicina. Universidade Estadual de Ciências da Saúde de Alagoas (UNCISAL), Maceió (AL), Brasil.

2. Acadêmico de enfermagem. UNCISAL, Maceió (AL), Brasil.

3. Professor adjunto e preceptor de estágio. UNCISAL, Maceió (AL), Brasil.

4. Professora titular. UNCISAL, Maceió (AL), Brasil.

$\triangle$ Claudio José dos Santos Júnior. Faculdade de Medicina (FAMED-UNCISAL). Rua Dr. Jorge de Lima, 113 - Trapiche da Barra. CEP: 57010-300. Maceió (AL), Brasil. claudiosantos_al@hotmail.com | Recebido: 17/02/2019 | Aprovado: 26/06/2019 


\section{INTRODUÇÃO}

O suicídio é um grande problema de saúde pública em todo o mundo e vem cada vez mais tendo destaque no Brasil ${ }^{1}$. Como fenômeno complexo, o suicídio constitui-se em um ato consciente, autodestrutivo, cometido por sujeitos em condições de vulnerabilidade emocional, mas que também podem ser potencializados por fatores sociais. O sujeito percebe o ato suicida como a melhor solução para fugir de situações que provocam uma dor psicológica insuportável².

$\mathrm{Na} 10^{\mathrm{a}}$ Classificação Internacional de Doenças (CID) o suicídio é identificado como uma violência de "causa externa", tendo uma categorização quanto à urgência, podendo ser baixa, média ou alta, a depender da ocorrência suicida. Além disso, o risco de suicídio pode partir de nenhuma perturbação até ideias de suicídio, podendo ou não estar associado com algum tipo de transtorno mental ${ }^{3}$.

No Brasil, cerca de 800 mil pessoas vêm a óbito em decorrência do suicídio todos os anos, sendo que para cada morte por suicídio ocorre uma média de vinte tentativas ${ }^{4}$. Segundo dados da Organização Mundial da Saúde (OMS) ${ }^{3}$, em 2014 o suicídio representou $1,4 \%$ de todas as mortes em todo o mundo, e em 2012 tornou-se a $15^{a}$ causa de mortalidade na população geral, sendo a segunda principal causa de morte entre os jovens de 15 a 29 anos. A OMS refere que a cada 40 segundos ocorre uma morte por suicídio e a cada três segundos, uma tentativa. A perspectiva é que em 2020 o número de mortes por suicídio alcançará cerca de 1,5 milhões de casos $^{5}$.

Diante da gravidade dos indicadores que apresentam um aumento significativo nos últimos anos, esse problema de saúde pública tornou-se tema de evento realizado pelo escritório da Organização Pan-Americana da Saúde/Organização Mundial de Saúde (OPAS/OMS), juntamente com o Ministério da Saúde, no último ano, colocando em pauta discussões referentes aos dados alarmantes, bem como a necessidade emergencial de ações preventivas, de proteção e promoção da vida ${ }^{6}$.

Faz-se necessário uma maior compreensão dos determinantes que envolvem o processo que resulta na tentativa e morte por suicídio, entendendo que intervenções eficientes realizadas por profissionais preparados para a assistência emergencial no risco e tentativa de suicídio, com ações voltadas para a sensibilização da sociedade de forma mais contextualizadas, sejam efetivas tanto na assistência ao indivíduo nos serviços de saúde quanto na prevenção deste agravo ${ }^{7}$.

É importante, também, a necessidade de pesquisas voltadas para a temática e público aqui apresentados, para que este campo de estudo possa avançar e contribuir cada vez mais com o processo de monitoramento e enfrentamento destes dados, com o intuito de contribuir para um melhor entendimento desse agravo, bem como, para a redução dos casos no estado de Alagoas.

Desta forma, o objetivo do presente estudo consiste em apresentar o perfil dos pacientes atendidos por tentativas de suicídio em um hospital geral de emergência do estado de Alagoas.

\section{METODOLOGIA}

Estudo do tipo documental, descritivo e retrospectivo com abordagem quantitativa. Foram selecionados os atendimentos hospitalares realizados entre o período de 1/1/2015 a 31/12/2017, codificados segundo a décima revisão da Classificação Internacional de Doenças e Problemas Relacionados à Saúde (CID-10) na categoria de lesões autoprovocadas intencionalmente (CID 10: X60-X84) no âmbito do Hospital Geral do Estado de Alagoas Dr. Oswaldo Brandão Vilela (HGE), uma unidade hospitalar pública de alta complexidade, referência em urgência, emergência e trauma, localizada em Maceió, Alagoas, Brasil.

A amostra da pesquisa foi todo o universo de pacientes ( $n=824)$ atendidos e diagnosticados pelas causas em estudo no HGE. As variáveis levantadas e analisadas foram: sexo (masculino; feminino); faixa etária (5 a 14 anos; 15 a 29 anos; 30 a 49 anos; 50 a 59 anos; 60 a 79 anos; $\geq 80$ anos; ignorado) e as categorias da CID-10 correspondentes ao suicídio, agrupadas neste estudo como: envenenamento (X60-X69); enforcamento e estrangulamento (X70); arma de fogo e objeto perfurocortante (X72-X74); objeto quente, fumaça, fogo e gás (X75-X77); arma branca e objetos contundentes (X78-X79); demais lesões autoprovocadas intencionalmente (X71, X8-84, X66-67). 
Realizou-se a descrição do perfil epidemiológico por meio de estatística descritiva dos dados, a partir da apuração de frequências simples absolutas e percentuais. Para tabulação, análise descritiva, cálculo de indicadores e construção de gráficos foi utilizado o programa Microsoft Office Excel 2010. Aplicou-se também o teste de regressão linear simples, a fim de se avaliar o padrão de crescimento (aumento, decréscimo ou constância) das ocorrências ao longo do período em estudo. A discussão dos achados foi realizada à luz da literatura especializada.

O banco de dados das notificações de atendimento foi disponibilizado através de protocolo n. ${ }^{\circ}$ 2216/2018 pela própria Instituição Hospitalar. Por se tratar de estudo realizado a partir de banco de dados secundários e de domínio público, sem a possibilidade de identificação de indivíduos, não foi necessária a submissão ao Comitê de Ética em Pesquisa, nem Termo de Consentimento Esclarecido, em atenção à Resolução CNS n. ${ }^{\circ}$ 510, de 7 de abril de 2016.

\section{RESULTADOS}

Neste estudo, a amostra investigada foi constituída de 824 usuários atendidos e diagnosticados por tentativa de suicídio no HGE.

No que diz respeito às características sociodemográfica dos pacientes (Tabela 1), obtivemos que $50,1 \%(n=413)$ dos pacientes têm a faixa etária entre 15 a 29 anos e $63 \%(n=522)$ são do sexo feminino.

Quanto à variável caracterização das lesões autoprovocadas por meio empregado (Tabela 2$), 82,1 \%(n=682)$ dos pacientes atendidos no hospital utilizaram o meio de envenenamento (X60-X69). Ao serem analisadas as maiores prevalências do tipo de envenenamento (Tabela 3 ), $58,9 \%(n=402)$ utilizaram o agente medicamento, $20,1 \%(n=137)$ raticida e $5,9 \%(n=40)$ produto de uso doméstico.

No estudo, verificou-se que a distribuição de ocorrências por atendimentos de suicídio entre 2015 a 2017 cresceu ao longo dos anos (Gráfico 1), fato que fica comprovado mediante linha de tendência crescente no teste de teste de regressão linear.

\section{Tabela 1}

Caracterização sociodemográfica dos pacientes atendidos por lesão autoprovocada no HGE, 2015-2017.

\begin{tabular}{lcc}
\hline Característica & $\mathrm{N}$ & $\%$ \\
\hline Faixa Etária & & \\
5 a 14 anos & 47 & 5,7 \\
15 a 29 anos & 413 & 50,1 \\
30 a 49 anos & 266 & 32,3 \\
5 a 59 anos & 48 & 5,8 \\
60 a 79 anos & 46 & 5,6 \\
80 anos e mais & 3 & 0,4 \\
Ignorado & 1 & 0,1 \\
Total & 824 & 100,0 \\
Sexo & & \\
Masculino & 302 & 36,7 \\
Feminino & 522 & 63,3 \\
Total & 824 & 100,0 \\
\hline
\end{tabular}

Fonte: Acervo documental do HGE (2018).

\section{Tabela 2}

Caracterização das lesões autoprovocadas por meio empregado em pacientes atendidos no HGE, 2015-2017.

\begin{tabular}{lcc}
\hline Meio utilizado / CID 10 & $\mathrm{N}^{*}$ & $\%$ \\
\hline $\begin{array}{l}\text { Envenenamento (X60-X69) } \\
\begin{array}{l}\text { Enforcamento e estrangulamento } \\
\text { (X70) }\end{array}\end{array}$ & 682 & 82,1 \\
$\begin{array}{l}\text { Arma de fogo e objeto } \\
\text { perfurocortante (X72-X74) }\end{array}$ & 69 & 8,3 \\
$\begin{array}{l}\text { Objeto quente, fumaça, fogo e gás } \\
\text { (X75-X77) }\end{array}$ & 21 & 2,5 \\
$\begin{array}{l}\text { Arma branca e objetos } \\
\text { contundentes (X78-X79) } \\
\begin{array}{l}\text { Outros meios de agressão } \\
\text { (X71, X8-84, X66-67) }\end{array}\end{array}$ & 21 & 2,5 \\
\hline Total & 18 & 2,2 \\
\hline Fonte: Acervo documental do HGE (2018). & 831 & 100 \\
\hline
\end{tabular}

*Tem-se um $\mathrm{N}$ superior ao número de atendimentos, pois em algumas ocorrências fora registrado pela equipe médica mais de um meio empregado na tentativa de suicídio.

O número de atendimentos por ocorrência de tentativa de suicídio foi maior, em termos absolutos, em alguns meses, no entanto, o teste de regressão linear indicou ausência de crescimento estatisticamente significativo entre meses do ano para o fenômeno em estudo (Gráfico 2).

Verificou-se que ambos os sexos apresentaram progressão no número de atendimentos absolutos (Gráfico 3), porém as mulheres lideraram os registros. 


\section{Tabela 3}

Caracterização das lesões autoprovocadas do tipo Envenenamento (CID 10 X60-X69) de acordo com o agente tóxico empregado em pacientes atendidos no HGE, 2015-2017.

\begin{tabular}{lcc}
\hline Agente & $\mathrm{N}$ & $\%$ \\
\hline Medicamento & 402 & 58,9 \\
Agrotóxico agrícola & 8 & 1,2 \\
Agrotóxico doméstico & 14 & 2,1 \\
Raticida & 137 & 20,1 \\
Prod. Veterinário & 7 & 1,0 \\
Prod. uso domiciliar & 40 & 5,9 \\
Cosmético & 3 & 0,4 \\
Prod. Químico & 12 & 1,8 \\
Metal & 1 & 0,1 \\
Drogas de abuso & 2 & 0,3 \\
Alimento e bebida & 1 & 0,1 \\
Outro & 6 & 0,9 \\
Ignorado & 49 & 7,2 \\
Total & 682 & 100,0 \\
\hline
\end{tabular}

Fonte: Acervo documental do HGE (2018).
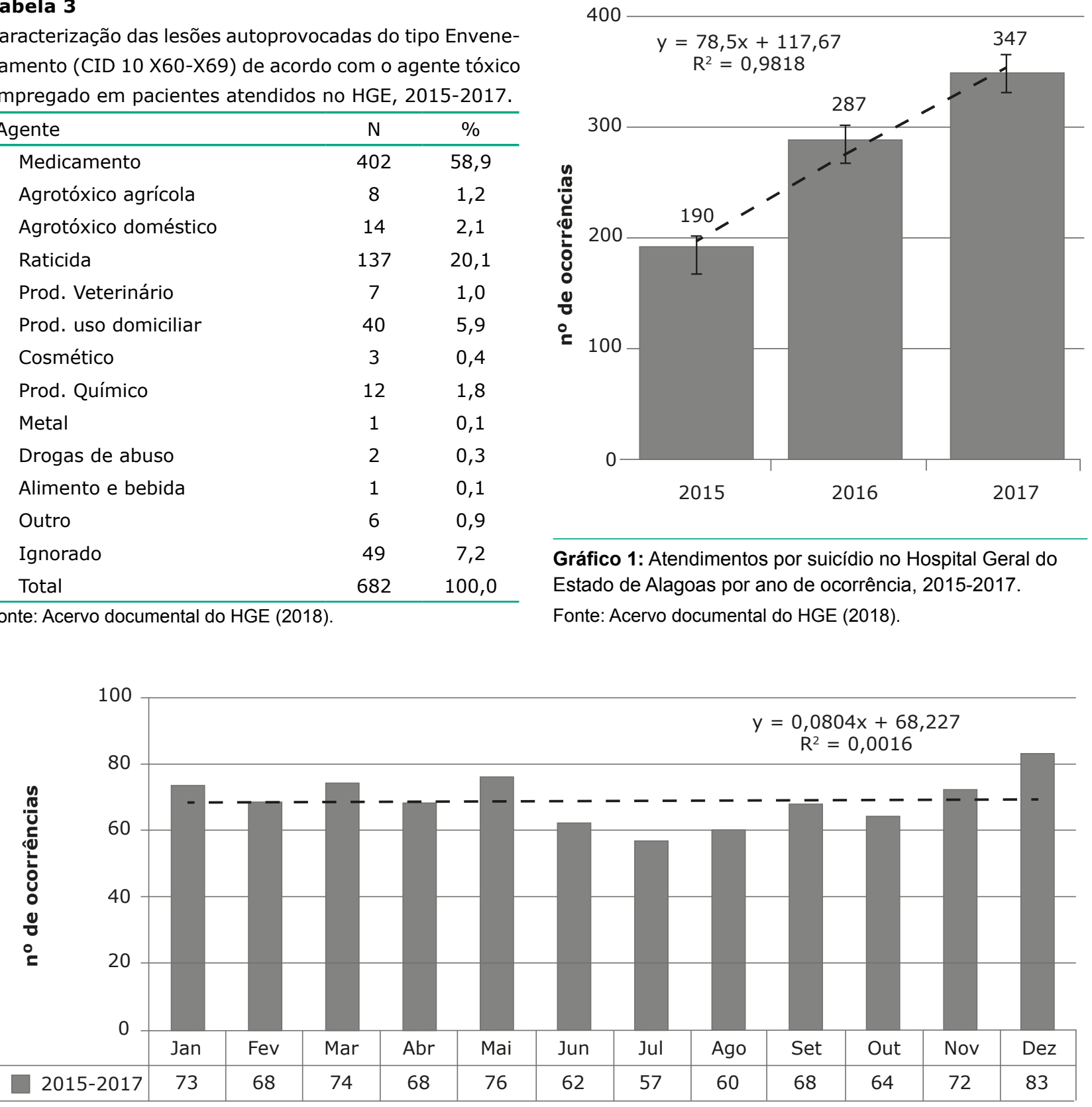

Gráfico 2: Atendimentos por suicídio no Hospital Geral do Estado de Alagoas por mês de ocorrência, 2015-2017. Fonte: Acervo documental do HGE (2018). 


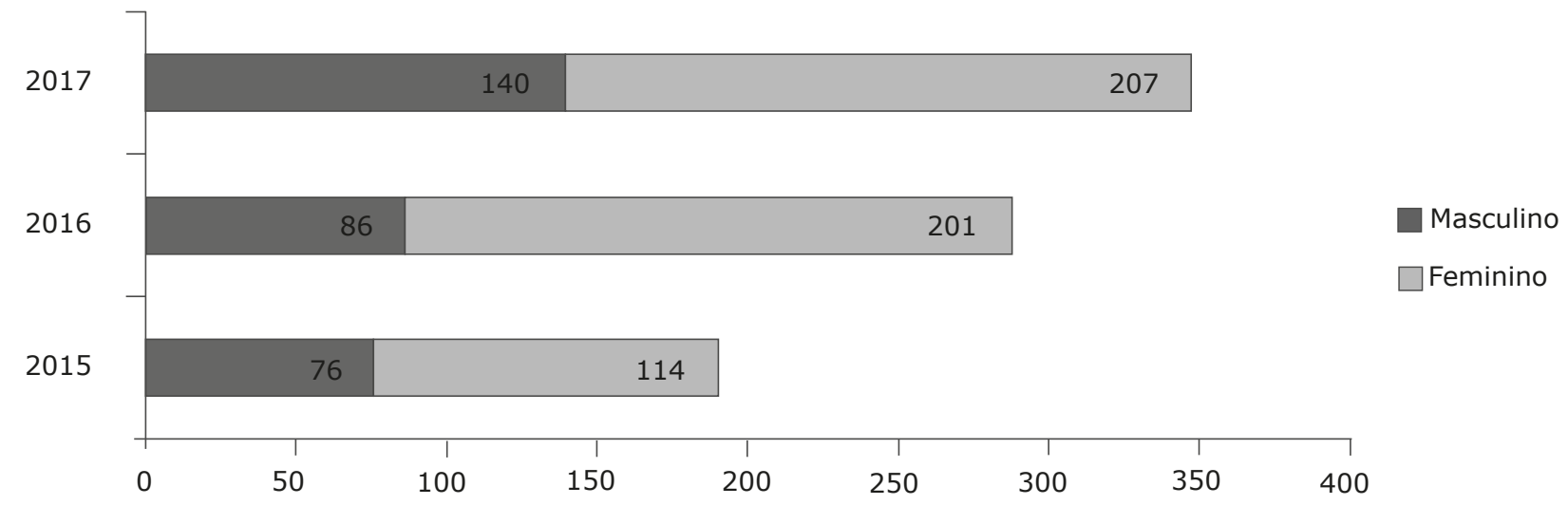

Gráfico 3: Atendimentos por suicídio no Hospital Geral do Estado de Alagoas segundo ano de atendimento e sexo do paciente, 2015-2017

Fonte: Acervo documental do HGE (2018).

\section{Tabela 4}

Atendimentos por suicídio no Hospital Geral do Estado de Alagoas por mês de ocorrência, 2015-2017.

\begin{tabular}{lcccc}
\hline Mês Ocorrência & 2015 & 2016 & 2017 & Total \\
\hline Janeiro & 15 & 27 & 31 & 73 \\
Fevereiro & 15 & 27 & 26 & 68 \\
Marco & 22 & 20 & 32 & 74 \\
Abril & 15 & 24 & 29 & 68 \\
Maio & 14 & 32 & 30 & 76 \\
Junho & 17 & 18 & 27 & 62 \\
Julho & 9 & 21 & 27 & 57 \\
Agosto & 14 & 24 & 22 & 60 \\
Setembro & 14 & 22 & 32 & 68 \\
Outubro & 16 & 19 & 29 & 64 \\
Novembro & 23 & 19 & 30 & 72 \\
Dezembro & 16 & 34 & 32 & 83 \\
Total & 190 & 287 & 347 & 824 \\
\hline
\end{tabular}

Fonte: Acervo documental do HGE (2018).

\section{DISCUSSÃO}

O suicídio é um problema global de saúde pública, pois compromete a vida dos indivíduos acarretando prejuízos psíquicos e sociais. $\mathrm{O}$ grupo onde há maior prevalência de tentativas de suicídio no Brasil é representado pelo sexo feminino, porém, o sexo masculino é identificado com maior efetividade no ato suicida de acordo com as notificações de atendimentos dos órgãos públicos de saúde ${ }^{3,6}$.
Os resultados presentes neste trabalho revelaram uma predominância de tentativas de suicídio no gênero feminino, demonstrando resultados similares à literatura nacional ${ }^{8,9}$. Diversos autores discutem que essa realidade se reflete pela maior frequência em utilização de ferramentas letais bruscas pelos indivíduos do sexo masculino, enquanto as mulheres se apropriam de métodos de menor invasão letal ${ }^{7,8}$.

Segundo a literatura especializada, a prevalência de tentativas de suicídio e o suicídio consumado tem um padrão sazonal, que é marcante no hemisfério norte, onde existe notável redução à exposição de luz solar nos meses mais frios ${ }^{10}$. Esta associação tem menor proporção perto da linha do equador, onde há pouca variação na luminosidade e insolação ao longo do ano ${ }^{9}$. Diversos trabalham relatam ainda que as mudanças climáticas e a poluição do ar estão associadas diretamente a problemas de saúde mental, como depressão e suicídio ${ }^{11}$. Confrontando os dados científicos, o presente estudo evidenciou não correlação sazonal entre as tentativas de suicídio atendidas no $H G E$, sugerindo que a variação no Estado de Alagoas, e possivelmente no Brasil, deve-se mais a fatores socioculturais, tendo provável relação com solidão, conflitos familiares, entre outros, do que propriamente as variações sazonais.

Corroborando com a literatura ${ }^{10}$, a ocorrência de tentativa de suicídio na puberdade é considerada rara. Em nosso estudo foi evidenciado que $5,7 \%(n=47)$ dos sujeitos com a faixa etária de 
5 a 14 anos foram atendidos por lesão autoprovocada. Os índices de tentativas aumentam com a idade, contudo, entre os jovens (15 a 29 anos), esses números permaneceram menos expressivos se comparados ao percentual de indivíduos na faixa etária adulta.

Entre os anos de 2010 a 2015, no Brasil, 448.499 casos de intoxicação por produtos químicos foram registrados. Destes, 33,2\% foram associados a tentativas de suicídio, aproximadamente 138.901 casos $^{12}$. Estudos atuais, realizados na Grécia, com 940 casos notificados, evidenciaram que a maior prevalência de tentativas ocorreu devido à overdoses de medicamentos ${ }^{13}$, corroborando com nossos estudos. Tal prevalência se refere ao hábito dos indivíduos possuírem estoques de medicamentos, fornecendo acesso à tentativas de suicídio, assim como ao suicídio ${ }^{14}$.

É curioso que a classe medicamentosa representada pelos benzodiazepínicos é predominante nas tentativas de suicídio ${ }^{15}$. Dos indivíduos que utilizam esses psicofármacos, $11,67 \%$ iniciaram o seu uso de forma autônoma e por indicação de amigos, já outros $40 \%$ foram prescritos por médico psiquiatra ${ }^{16,17}$. Uma limitação deste trabaIho é a ausência de especificação da classe de medicamentos utilizadas na tentativa de suicídio, limitação essa fruto da ausência desse tipo de registro na fonte de dados empregada.

No presente estudo, o uso de raticida para tentativas de suicídio $(20,1 \%)$ foi maior, comparando com outros tipos de agrotóxico domésticos e agrícolas, corroborando com a literatura sobre o tema ${ }^{18}$

Verificou-se que os casos de suicídio têm crescido ao longo dos anos e que a rede de saúde local tenta, segundo as suas possibilidades, assistir à população, monitorando os atendimentos e as notificações de ocorrência do problema. Os hospitais, como é o caso da unidade de saúde objeto desta investigação, são instituições de grande abrangência social no território em que estão inseridas, com a função principal de receber a maior demanda de casos graves, sendo eles de suicídio ou de diversos problemas, exercendo um papel notificador desses casos ${ }^{7}$.

A detecção de fatores de risco para o suicídio já é relatada, à luz da literatura, como método de prevenção e intervenção. Diante disso, é crucial identificar pacientes com nova tentativa de suicídio para que sejam implementadas intervenções mais intensas, assim como o controle do tratamento medicamentoso ${ }^{19}$.

Importante destacar, ainda, algumas das ferramentas estratégicas que os gestores e profissionais da saúde podem utilizar na tentativa de reduzir a ocorrência do agravo, objeto de análise neste estudo, são elas: promover a consciência de que o suicídio é um grande problema de saúde e que ele pode ser prevenido; desenvolver e ampliar a base de apoio para prevenção de suicídio; desenvolver e programar estratégias para minimizar os estigmas associados a ser usuário de saúde mental; e promover esforços para reduzir o acesso a meios e métodos letais de danos a si mesmo.

A unidade hospitalar objeto deste estudo é pioneira no Brasil em notificar casos de violência autoprovocada. Antes mesmo da Lei Federal 13.819, sancionada em 26 de abril de 2019, que criou a Política Nacional de Prevenção da Automutilação e do Suicídio, a referida instituição já notificava os casos de tentativa de suicídio em sistema de informação próprio. O texto da Lei Federal 13.819/2019 dispõe que a violência autoprovocada pode ser caracterizada pelo suicídio consumado, pela tentativa de suicídio e pelo ato de automutilação, com ou sem idealização suici$\mathrm{da}$, e que todas essas situações devem ser notificadas de forma compulsória pelos estabelecimentos de saúde ${ }^{20}$.

\section{CONCLUSÃO}

Analisando os dados obtidos, nota-se que houve predomínio de tentativas de suicídio em pacientes com idades entre 15 a 29 anos e do sexo feminino, utilizando como meio o envenenamento do tipo medicamentoso. Houve ainda tendência de crescimento no número de ocorrências do agravo nos anos em estudo.

Diante do exposto, o presente estudo fornece informações importantes para a saúde pública do estado de Alagoas, cuja contribuição pode permitir e incentivar a promoção da saúde e a prevenção desse dano à população. 
O estudo é ainda relevante, pois traz um cenário inédito das tentativas de suicídio em uma unidade pioneira no Brasil em notificar casos de violência autoprovocada, antes mesmo de ser estabelecida a obrigatoriedade de notificação através pela Lei Federal 13.819 de 2019.

\section{Conflito de Interesses}

Não há.

\section{Fonte de fomento}

Não há.

\section{Aprovação do comitê de ética em pes- quisa}

Não se aplica.

\section{Registro dos ensaios clínicos em base de acesso público}

\section{Não se aplica.}

\section{REFERÊNCIAS}

1. Vidal CEL, Gontijo ED. Tentativas de suicídio e o acolhimento nos serviços de urgência: a percepção de quem tenta. Cad Saúde Colet. 2013;21(2):108-14.

2. Sousa GS, Silva RM, Figueiredo AEB, Minayo MCS, Vieira LJES. Circunstâncias que Envolvem o Suicídio de Pessoas Idosas. Interface (Botucatu, Online). 2014;18(49):1-13.

3. Ribeiro NM, Castro SDE, Scatena LM, Haas VJ. Análise da Tendência temporal do suicídio e de Sistemas de Informações em Saúde em Relação às Tentativas de Suicídio. Texto Contexto Enferm. 2018;27(2):e2110016.

4. Brasil, Suicídio. Saber, Agir e Prevenir. Secretaria de Vigilância em Saúde - Ministério da Saúde. Volume 48 Nº 30 [internet] 2017 [cited $2018 \mathrm{dec} 15$ ]. Available from: http://portalarquivos2.saude.gov.br/images/pdf/2017/ setembro/21/2017-025-Perfil-epidemiologico-das-tentativas-e-obitos-por-suicidio-no-Brasil-e-a-rede-de-atencao-a-saude.pdf

5. Rosa NM, Agnolo CMD, Oliveira RR, Mathias TAF. Tendência de declínio da taxa de mortalidade por suicídio no Paraná, Brasil: contribuição para políticas públicas de saúde mental. J Bras Psiquiatr. 2017;66(3):157-63

6. Brasil, Organização Pan-Americana da Saúde/Organização Mundial da Saúde. [internet] [cited 2018 nov 01]. Disponível em: https://www.paho.org/bra/index. php?option $=$ com_content\&view $=$ article\&id $=5674$ : suici dio-e-grave-problema-de-saude-publica-e-sua-prevencao-deve-ser-prioridade-afirma-opas-oms\&Itemid = 839

7. Santos EGO, Oliveira YOMCO, Azevedo UM, Nunes ADS, Amador AE, Barbosa IR. Análise espaço-temporal da mortalidade por suicídio em idosos no Brasil. Rev Bras Geriatr. Gerontol. 2017;20(6):854-65.

8. Fiocruz/CICT/Sinitox. Fundação Oswaldo Cruz/Science and Technology Research Centre/Toxic-Pharmacologic Information National System. Annual Statistic of Poisoning and Intoxication Occurrences. Brazil. [cited 2018 dez 15]. Disponível em: http://www.fiocruz.br/sinitox/ cgi/cgilua.exe/sys/start.htm?sid=8.

9. Bauer M, Glenn T, Alda M, Andreassen OA, Angelopoulos E, Ardau $\mathrm{R}$, et al. Association between solar insolation and a history of suicide attempts in bipolar I disorder. J Psychiatr Res [internet]. 2019 [acesso em 19 ago 2019];113:1-9. doi: https://doi: 10.1016/j.jpsychires.2019.03.001

10. Gładka A, Rymaszewska J, Zatoński T. Impact of air pollution on depression and suicide. Int J Occup Med Environ Health [internet]. 2018 [acesso em 19 ago 2019];31(6):711-21. doi: https://doi.org/10.13075/ ijomeh.1896.01277

11. Sadock BJ, Sadock VA, Ruiz P. Compêndio de psiquiatria: ciência do comportamento e psiquiatria clínica. Porto Alegre: Artmed; 2017.

12. The National System of Aggravations and Notification (Sinan). Intoxicação Exogéna. [cited 2018 dez 15]. Disponível em: http://www2.datasus.gov.br/DATA-SUS/index. php?area $=0203 \&$ id $=29878153$

13. Fountoulakis KN, Savopoulos C, Apostolopoulou M, Dampali R, Zaggelidou E, Karlafti E, et al. Rate of suicide and suicide attempts and their relationship to unemployment in Thessaloniki Greece (2000-2012). J Affect Disord [internet]. 2015 [acesso em 19 ago 2019];174:131-6. doi: 10.1016/j.jad.2014.11.047

14. Santos SA, Legay LF, Lovisi GM, Santso GFC, Lima LA Suicídios e tentativas de suicídios por intoxicação exógena no Rio de Janeiro: análise dos dados dos sistemas oficiais de informação em saúde, 2006-2008. Rev Bras Epidemiol. 2013;16(2):376-87.

15. Muheim F, Martin E, Pascal B, Stephanie C, Gabriela S, Merle $\mathrm{K}$, et al. Suicide attempts in the county of Basel: results from the WHO/EURO Multicentre Study on Suicidal Behaviour. Swiss Med Wkly. 2013;143:w13759.

16. Konopka A, Pełka-Wysiecka J, Grzywacz A, Samochowiec J. Psychosocial characteristics of benzodiazepine addicts compared to not addicted benzodiazepine users. Prog neuropsychopharmacol biol psychiatr. 2013;40:229-35.

17. Liotta M, Mento C, Settineri S. Seriousness and lethality of attempted suicide: A systematic review. Aggression and Violent Behavior. 2015;21:97-109. 
18. Almeida TSO, Fook SML, Mariz SR, Camêlo ELS, Gomes LCF. Suicide attempts: epidemiologic trends towards geoprocessing. Ciênc saúde coletiva [internet]. 2018 [acesso em 19 ago 2019];23(4):1183-92. doi: http:// dx.doi.org/10.1590/1413-81232018234.12452016

19. Espandian A, Gonzálen M, Reijas T, Florez G, Ferrer E, Saiz PA, et al. Relevant risk factors of repeated suicidal attempts in a sample of outpatients. Rev Psiquiatr Sa- lud Ment [internet]. 2019 [acesso em 19 ago 2019];pii: S1888-9891(19):30047-3. doi: https://doi: 10.1016/j. rpsm.2019.03.003.

20. Alves T. HGE é pioneiro no Brasil em notificar casos de violência autoprovocada. Agência Alagoas. Disponível em: http://agenciaalagoas.al.gov.br/noticia/item/30136-hge-e-pioneiro-no-brasil-em-notificar-casos-de-violencia-autoprovocada. Acesso em: 23 de junho de 2017 